\title{
Aligning Performance Measurement and Practice Trends for Developmental Delay Screening
}

Knapp C*, Baker K and Baron-Lee J

Department of Health Outcomes and Policy, Institute for Child Health Policy, University of Florida, Gainesville, FL, USA

*Corresponding author: Caprice Knapp, Department of Health Outcomes and Policy, Institute for Child Health Policy, 1329 SW 16 th Street, Gainesville, FL 32610, USA, Tel: 3522652517; E-mail: Caprice1@ufl.edu

Received date: Dec 22, 2015; Accepted date: Feb 26, 2016; Published date: Mar 11, 2016

Copyright: ( 2016 Knapp C, et al. This is an open-access article distributed under the terms of the Creative Commons Attribution License, which permits unrestricted use, distribution, and reproduction in any medium, provided the original author and source are credited.

\section{Introduction}

In the United States, it is estimated that nearly $20 \%$ of children under the age of 18 experience developmental delays (DDs) [1]. Children with DDs are often affected in physical, cognitive, communicative, and, or, social domains, and frequently need special education [2]. Appropriate screening, early diagnoses, and timely interventions for these children can increase the likelihood of overcoming such delays and improving their outcomes. Studies have shown that children who receive early intervention are more likely to complete secondary school, have better job placements, have higher incomes, attend college, and have children at an older age as compared to their peers with DD who do not receive early intervention [3]. Additionally, early intervention reduces long term effects of DDs, improves areas of difficulty after diagnosis, and for chronic delays, maximizes overall function and seeks to increase independence [4].

Although the benefits of early screening have been well described in the literature, challenges continue to exist, among pediatricians, in determining and using appropriate and valid DD screening tools. Moreover, these challenges exist in spite of evidence that standard DD screening is beneficial [5]. Typically, pediatricians do assess for DDs but do not always use standardized screening tools. Pediatricians often report that clinical impressions and nonstandard forms are used yet evidence shows this leads to misdiagnoses of DDs.5 Common barriers to standard screening include lack of time, limited reimbursement, and insufficient training using and understanding standard tools [5].

This brief article is written to highlight current practice trends of DD screening and how they need to be better aligned with performance measurement expectations. We address the importance of standard DD screening, the need for integrating current practices of developmental screening with required standard reporting, the idea that pediatricians' behaviors can be changed to accommodate standard reporting requirements, and factors that may affect standard measurement use. Ultimately, the goal is to address and overcome these challenges and to ensure that all children who need a DD screening receive one.

Keywords: Developmental; Screening; Children

\section{Importance of screening}

Screening for DDs using standard measures is a critical aspect of improving outcomes for children with DDs. Though clinical impressions of DDs are important, there is a greater need for using standard measures of developmental assessment. Non-standard developmental screening measures may have proved sufficient in the past; however, pediatricians must now strive to improve their use of standard DD screening tools. The use of standardized tools has several benefits including allowing for 1) comparison of rates and variations in DD screening across the nation, 2) tracking of DD trends over time, 3) improved outcomes for children with DD, 4) increased parent satisfaction, and 5) potentially reduced effort and time from the paediatrician [6].

Assessment of DD screening has been highlighted by professional organizations and most recently, through inclusion as a measure in the Children's Health Insurance Program Reauthorization Act (CHIPRA) core measure set. The CHIPRA legislation has many parts designed to improve health and health care for children in the United States, including annual voluntarily reporting on 24 core measures to the Centers for Medicare and Medicaid Services. Core measures were chosen based on their evidence, reliability, and validity. The Developmental Screening in the First Three Years of Life measure was included in the core set.

Compliance for this measure requires medical record review and evidence that one of the following six standardized tools were used: 1) Ages and Stages Questionnaire, 2) Infant Development Inventory, 3) Child Development Inventory, 4) Bayley Infant Neurodevelopmental Screener, 5) Parents' Evaluation of Developmental Status, or 6) Brigance Screen-II, and Battelle Developmental Inventory. Currently, there are no state or national benchmarks for this measure.

\section{Reconciling practice trends and expectations}

Since 2006, our team has conducted approximately 500 medical record reviews per year for the purposes of determining compliance with a number of quality of care measures. In our reviews it is clear that pediatricians are documenting developmental milestones of young children, yet the majority are not using any of the previously mentioned standard tools [7]. Instead, most pediatric charts that we review show that clinical milestones or general notes on development are made.

This implies that pediatricians deem developmental assessment essential to conduct and document. It also suggests that pediatricians' behaviors may be modified if they can be convinced to use that time to utilize standard tools in place of notes or rudimentary checklists. How might pediatricians' behaviors be modified? Reimbursement is one method that can be used. For instance, in Illinois pediatricians are receiving financial incentives for compliance with standard DD screenings. Other contracted financial arrangements based on DD performance measurement results can be used to reward compliance as well. Pediatricians' behaviors can also be modified in ways beyond using financial incentives. For example, adjusting the curriculum of medical students and residents to not only understand the importance of DD screening, but also focus on the use of standardized DD tools can also impact behaviors. In addition, pediatric students and residents 
should be trained on how to interpret results from standardized tools and follow up as needed through referrals to specialists or early intervention programs. Quality improvement initiatives, such as the Assuring Better Child Health and Development (ABCD) initiative, have also been used to increase screenings. Regardless of how stakeholders aim to modify practice trends, it is important to bridge the gap between the current state of screening and local, state, and national expectations.

\section{Factors that affect screening}

Further research also needs to be conducted to understand the factors that affect standard DD screening. In order to appropriately screen for DDs, pediatricians need to be mindful that several implicit and explicit factors may influence DD screening. Again, in our work we have noted that that child and health care delivery characteristics seem to effect whether DD screenings are done. For example, our data agreed with national screening trends which show that male children are twice as likely to have a DD as females. However, pediatricians still need to appropriately screen female children for DDs to avoid having a disproportionate screening for male children.

In addition, in our data non-minority children tend to be screened for DDs more frequently than minorities. Understanding how these and other factors affect DD screening, will best help target subgroups with lower levels of compliance.

\section{Conclusion}

Key challenges continue to exist as barriers to using standard DD screening tools. Beyond cited challenges such as lack of time, limited or no compensation, and limited training, there are additional challenges related to a lack of systematic screening and the reluctance to switching from existing non-standard methods of assessment. However, using standard DD screening tools may actually reduce the amount of time a pediatrician exerts since knowledge of the tool and improvements in using the tool will be made over time. Other strategies to overcoming challenges to using standard DD measures include outreach by health plan provider liaisons, campaigns by community organizations, and educational materials that empower parents to inquire about screening. Financial incentives and public reporting have both been found to be effective in altering provider behaviors and may also be necessary to overcoming challenges to using standard DD screening tools.

Screening for DDs using standard tools is a critical first step in identifying children that require treatment. Several professional organizations have already endorsed early and continuous screening for DDs using standard tools. Recently, the CHIPRA legislation included measures to understand compliance with DD screening. As regular reporting continues, it will be easier to determine variations across the nation and to track trends over time which may address external factors and barriers that affect standard DD tools.

\section{References}

1. (2001) Developmental surveillance and screening of infants and young children. Pediatrics 108: 192-196.

2. (2004) Individuals with Disabilities Education Improvement Act of 2004. Public Law 108-446.

3. Chamberlain RW (1987) Developmental assessment and early intervention programs for young children: Lessons learned from longitudinal research. Pediatr Rev 8: 237-247.

4. Majnemer A (1998) Benefits of early intervention for children with developmental disabilities. Semin Pediatr Neurol 5: 62-69.

5. Sices L (2007) Developmental Screening in Primary Care: The Effectiveness of Current Practice and Recommendations for Improvement. Washington, DC: The Commonwealth Fund.

6. Pinto-Martin JA, Dunkle M, Earls M, Fliedner D, Landes C (2005) Developmental stages of developmental screening: steps to implementation of a successful program. Am J Public Health 95: 1928-1932.

7. Knapp CA, Wang H, Baker K, Ralston D (2012) Pediatric performance measure in Florida's CHIPRA demonstration grant. 